Special issue of the 2nd International Conference on Computational and Experimental Science and Engineering (ICCESEN 2015)

\title{
Effect of Different $S U(3)$ Color Representations on the Nucleation of Quark-Gluon Plasma Droplets from a Hadronic Gas
}

\author{
K. Mezouar* and A. Ait El Djoudi \\ Laboratoire de Physique des Particules et Physique Statistique, Ecole Normale Supérieure, \\ Vieux-Kouba, B.P. 92, 16050, Algiers, Algeria
}

\begin{abstract}
We study the nucleation of quark-gluon plasma (QGP) droplets from the hadronic gas phase, based on the calculation of the difference in free energy in the QGP and hadronic gas phases. We investigate this difference in free energy by modeling it in different cases, when the surface tension of the QGP bubble is considered or not. We calculate the free energies of both hadronic gas consisting of massive pions and plasma droplet with up, down and strange quarks additionally to gluons, for different $S U(3)$ color representations of the QGP, namely the color singlet, color octet, and color 27-plet. The behavior of the obtained change in free energy is examined with varying radius of the QGP bubble, and at different temperatures. The effect of the surface contribution on the nucleated bubble is also investigated and compared to the case without this contribution.
\end{abstract}

DOI: 10.12693/APhysPolA.130.202

PACS/topics: 12.38.Mh, 64.60.Qb, 2.20.-a, 5.70.Ce

\section{Introduction}

The description of strong interactions by quantum chromodynamics (QCD) imposes the confinement of quarks into hadrons. However, lattice QCD calculations predict a dissolution of hadrons into quarks and gluons for high temperatures and/or densities. In these conditions, nuclear matter could thus exist in a novel state called the "Quark Gluon Plasma" (QGP). For several years, experiments have attempted to sign the formation of this plasma, and to characterize and study this novel state of nuclear matter.

The energy densities achieved during central collisions of large nuclei at Brookhaven National Laboratory's AGS may be high enough to allow the creation of the QGP [1]. This formation of the QGP state of matter may proceed through the nucleation of QGP droplets in hot hadronic gas (HG). In this case, the fields in the plasma obey the bag boundary conditions, staying inside the plasma droplet [2].

The nucleation process is driven by statistical fluctuations, generating quark-gluon plasma bubbles in the hot hadronic phase $[1,3,4]$. According to the phenomenological approach to the classical theory of nucleation formulated by Becker and Döring [5], and Frenkel [6], these fluctuations are determined by the change in free energy between the HG and QGP phases [3, 4].

In the aim of studying this process, we calculate in the present work the difference in free energy of the hadronic gas with massive pions and the QGP droplets. These later are taken first to consist of gluons, massless up and down quarks and their antiquarks, and in a second case, we take the massive strange quarks additionally to gluons

\footnotetext{
* corresponding author; e-mail: mmezouar@yahoo.fr
}

and massless $u$ and $d$ quarks. The calculation of the free energy of QGP bubbles is effectuated for different $S U$ (3) color group representations namely the color singlet, color octet and color 27-plet. We study the behavior of the obtained change in free energy with varying radius of the nucleated QGP bubble, at different temperatures, and for different values of surface tension.

\section{Change in free energy for different color representations of the QGP droplet}

In the bag model, the variation in free energy during the QGP droplet formation in the hot hadronic gas, representing the free energy barrier which has to be surmounted by the nucleated bubble, has the general form given in [1] and can be written in its simplest form as:

$$
\Delta F=V\left(P_{\mathrm{HG}}-P_{\mathrm{QGP}}\right)+B V+4 \pi R^{2} \sigma,
$$

where $V$ is the volume of the formed plasma droplet, which is supposed to be spherical of radius $R\left(V=\frac{4}{3} \pi R^{3}\right), B$ is the bag constant, $\sigma$ is the surface tension and $P_{\mathrm{QGP}}, P_{\mathrm{HG}}$ are the pressures of the plasma droplet and the hadronic gas respectively. These pressures are calculated by the well known relation:

$$
P_{i}=T \frac{\partial \ln Z_{i}}{\partial V}
$$

where $Z_{i}$ represents the partition function of the QGP state or the hadronic state, and $T$ is the system temperature.

For the HG phase, we calculate the partition function $Z_{\mathrm{HG}}$ for massive pions gas, and the deduced pressure is given by:

$$
P_{\mathrm{HG}}=\frac{1}{2 \pi^{2}} \int_{0}^{\infty} \frac{k^{4} d k}{\sqrt{k^{2}+m_{\pi}^{2}}\left(\mathrm{e}^{\beta \sqrt{k^{2}+m_{\pi}^{2}}}-1\right)},
$$

where $\beta$ is the inverse temperature, $k$ the momentum and $m_{\pi}$ the pion mass. 
For the QGP, using the group theoretical projection method, formulated by Turko and Redlich [7], we calculate the partition function $Z_{\mathrm{QGP}}$ for a QGP containing two quark flavors (massless up and down) or three quark flavors (massless $u, d$ and massive $s$ ) in addition to antiquarks and gluons. Such a calculation has been carried out for a color singlet representation in the case of two massless $u$ and $d$ quarks in the previous works [8, 9] of one of us. In the present work, we calculate $Z_{\mathrm{QGP}}$ for different $S U$ (3) color representations: color singlet, color octet and color 27-plet. The general form of the obtained partition function of the QGP can be written as:

$$
Z_{\mathrm{QGP}}(T, V, \mu)=\frac{8 d(p, q)}{3 \pi^{2}} \int_{-\pi}^{+\pi} \int_{-\pi}^{+\pi} \mathrm{d}\left(\frac{\varphi}{2}\right) \mathrm{d}\left(\frac{\theta}{3}\right) M(\varphi, \theta) \chi^{*}(p, q) \mathrm{e}^{V T^{3} g_{1}\left(\varphi, \theta, \frac{\mu}{T}\right)+R T g_{2}\left(\varphi, \theta, \frac{\mu}{T}\right)+g_{3}\left(\varphi, \theta, \frac{\mu_{S}}{T}, m_{S}, R, T\right)},
$$

with:

$$
\begin{gathered}
g_{1}\left(\varphi, \theta, \frac{\mu}{T}\right)=\frac{\pi^{2}}{12}\left[2 d_{\mathrm{Q}} \sum_{q=r, g, b}\left(\frac{7}{30}-\left(\frac{\alpha_{q}-i\left(\frac{\mu}{T}\right)}{\pi}\right)^{2}+\frac{1}{2}\left(\frac{\alpha_{q}-i\left(\frac{\mu}{T}\right)}{\pi}\right)^{4}\right)\right. \\
\left.+d_{\mathrm{G}} \sum_{g=1}^{4}\left(-\frac{7}{30}+\left(\frac{\alpha_{g}-\pi}{\pi}\right)^{2}-\frac{1}{2}\left(\frac{\alpha_{g}-\pi}{\pi}\right)^{4}\right)\right], \\
g_{2}\left(\varphi, \theta, \frac{\mu}{T}\right)=\frac{2 \pi}{3}\left[\frac{d_{\mathrm{Q}}}{2} \sum_{q=r, g, b}\left(\left(\frac{\alpha_{q}-i\left(\frac{\mu}{T}\right)}{\pi}\right)^{2}-\frac{1}{3}\right)-d_{\mathrm{G}} \sum_{g=1}^{4}\left(-\frac{1}{3}+\left(\frac{\alpha_{g}-\pi}{\pi}\right)^{2}\right)\right], \\
g_{3}\left(\varphi, \theta, \frac{\mu_{S}}{T}, m_{S}, R, T\right)=d_{\mathrm{Q}}\left[\frac{V}{\sqrt{2} \pi^{\frac{3}{2}}}\left(m_{S} T\right)^{\frac{3}{2}}+\left(R^{2}+\frac{R}{m_{S}}\right)\left(\sqrt{\frac{2}{\pi}}\left(m_{S} T^{3}\right)^{\frac{1}{2}}-m_{S} T\right)\right. \\
\left.+\frac{2}{3} \sqrt{\frac{2}{\pi}} R\left(m_{S} T\right)^{\frac{1}{2}}\right] \mathrm{e}^{-\frac{m_{S}}{T}} \sum_{q=r, g, b}\left(1+\frac{\left(\frac{\mu_{S}}{T}+i \alpha_{q}\right)^{2}}{2 !}+\frac{\left(\frac{\mu_{S}}{T}+i \alpha_{q}\right)^{4}}{4 !}\right),
\end{gathered}
$$

where $d(p, q)$ and $\chi(p, q)$ are the dimension and the character of the $S U$ (3) color representation, respectively, and $M(\varphi, \theta)$ the Haar measure, which can be found in [10]. $\mu$ is the chemical potential for massless $u$ and $d$ quarks and $\mu_{S}$ that for $s$ quarks of mass $m_{S}, d_{\mathrm{Q} / \mathrm{G}}$ the spinisospin degeneracy factors for quarks and gluons, and $\alpha_{q}(q=r, g, b), \alpha_{g}(g=1, \ldots, 4)$ the angles determined by the eigenvalues of the color isospin and the color hypercharge operators, given by [11]:

$$
\begin{gathered}
\alpha_{r}=\frac{\varphi}{2}+\frac{\theta}{3}, \quad \alpha_{b}=\frac{-2 \theta}{3}, \quad \alpha_{g}=\frac{-\varphi}{2}+\frac{\theta}{3}, \\
\alpha_{1}=\alpha_{r}-\alpha_{g}, \quad \alpha_{2}=\alpha_{g}-\alpha_{b}, \quad \alpha_{3}=\alpha_{b}-\alpha_{r}, \\
\alpha_{4}=0 .
\end{gathered}
$$

Once the expressions of the QGP partition functions in the different color representations (singlet, octet, and 27-plet) are obtained, we then numerically calculate the change in free energy $\Delta F$ from Eq. (1), for each color representation of the QGP with massless $u$ and $d$ quarks, as well as with three quark flavors (massless up and down and massive strange quarks). The calculus is done at a vanishing chemical potential, with the common value $B^{1 / 4}=200 \mathrm{MeV}$. We then study the variation of $\Delta F$ by plotting it as a function of the radius of the plasma droplet at different temperatures and for various values of the surface tension.

\section{Results and discussion}

Our results for the variations of the free energy difference of the plasma bubble, with two and three quark flavors, as a function of the radius of the plasma droplet, are represented in Fig. 1, for different color representations, with vanishing surface tension $\sigma=0$, and at the temperature $T=150 \mathrm{MeV}$. This figure shows that the radius of the QGP droplet formed in the nucleation process, corresponding to a maximum of $\Delta F$, depends both on the number of quark flavors and on the color representation. This radius takes the smallest value for the color 27-plet representation, a higher value for the color octet representation and the highest value for the color singlet representation. And for each color representation, the bubble radius for the QGP with $u, d$ quarks corresponding to a maximum of $\Delta F$ is higher than the bubble radius maximizing $\Delta F$ for the QGP with $u, d$ and $s$ quarks.

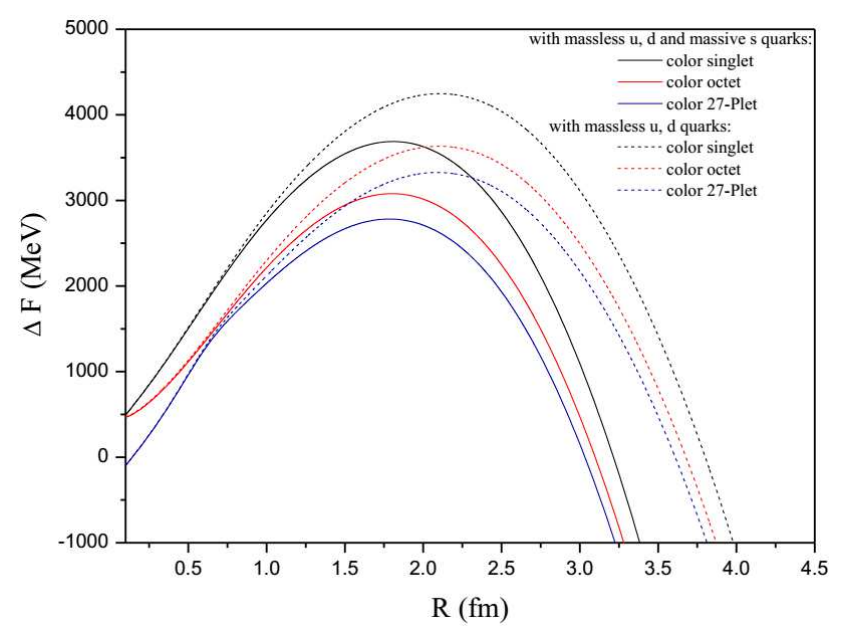

Fig. 1. Change in free energy vs QGP droplet radius with vanishing surface tension, at the temperature $T=150 \mathrm{MeV}$ and for the two cases of two quark flavors (dashed lines) and three quark flavors (solid lines), in the three color representations: color singlet, color octet, color 27-plet. 
The effect of the surface tension on the nucleation of the QGP bubble from a hadronic gas is also investigated, and Fig. 2 illustrates the change in free energy for two more values of the surface tension: $\sigma=20 \mathrm{MeV} / \mathrm{fm}^{2}$ (top) and $\sigma=30 \mathrm{MeV} / \mathrm{fm}^{2}$ (bottom) at the temperature $T=150 \mathrm{MeV}$.

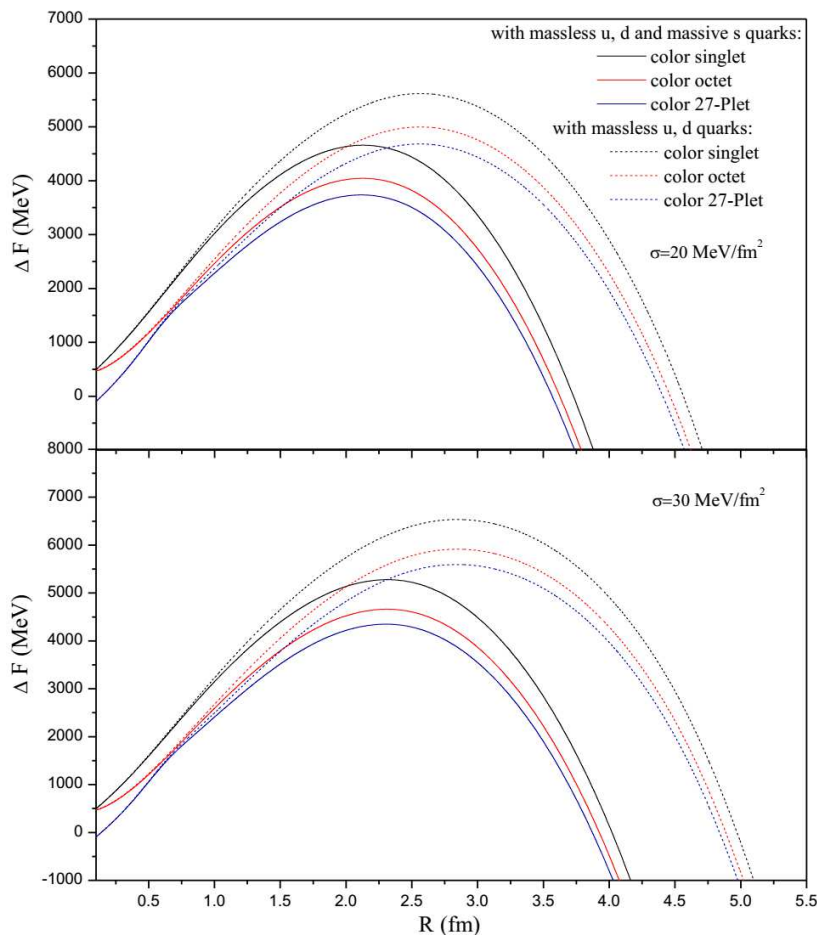

Fig. 2. Change in free energy vs QGP droplet radius at $T=150 \mathrm{MeV}$, with two surface tension values: $\sigma=20 \mathrm{MeV} / \mathrm{fm}^{2}$ (top), $\sigma=30 \mathrm{MeV} / \mathrm{fm}^{2}$ (bottom) and for the two cases of two quark flavors (dashed lines) and three quark flavors (solid lines), in the three color representations: color singlet, color octet, color 27-plet.

Additionally to the features previously observed in Fig. 1, which can also be noted for these two cases with $\sigma \neq 0$, another characteristic which can be drawn is that the radius of the nucleated QGP droplet, corresponding to a maximum of $\Delta F$, for the same color representation, increases when the surface tension increases. This can be seen more clearly in Fig. 3, which illustrates the variations of the change in free energy with the radius of the QGP droplet, in the color singlet representation with two massless $u$ and $d$ quarks, at the temperature $T=150 \mathrm{MeV}$. The radius maximizing $\Delta F$ is $R_{m}=2.11 \mathrm{fm}$ for $\sigma=0, R_{m}=2.57 \mathrm{fm}$ for $\sigma=20 \mathrm{MeV} / \mathrm{fm}^{2}$ and $R_{m}=2.85 \mathrm{fm}$ for $\sigma=30 \mathrm{MeV} / \mathrm{fm}^{2}$.

Additionally, and in the aim of studying the effect of the temperature on the variation of the change in free energy with QGP droplet radius, $\Delta F$ is plotted in Fig. 4 with $\sigma=20 \mathrm{MeV} / \mathrm{fm}^{2}$ and at two temperatures $T=$ $150 \mathrm{MeV}$ and $T=160 \mathrm{MeV}$ for a QGP with massless $u$, $d$ quarks (bottom) and with massless $u$ and $d$ and massive $s$ (top) in the three color representations.

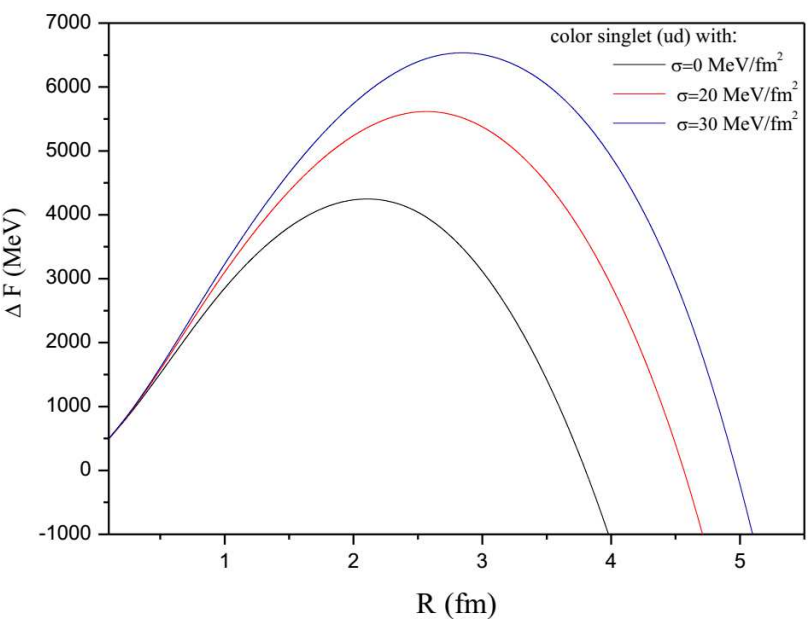

Fig. 3. Change in free energy vs the radius of QGP droplet with two quark flavors, at the temperature $T=$ $150 \mathrm{MeV}$ and with three surface tension values: $\sigma=0$, $\sigma=20 \mathrm{MeV} / \mathrm{fm}^{2}$ and $\sigma=30 \mathrm{MeV} / \mathrm{fm}^{2}$.

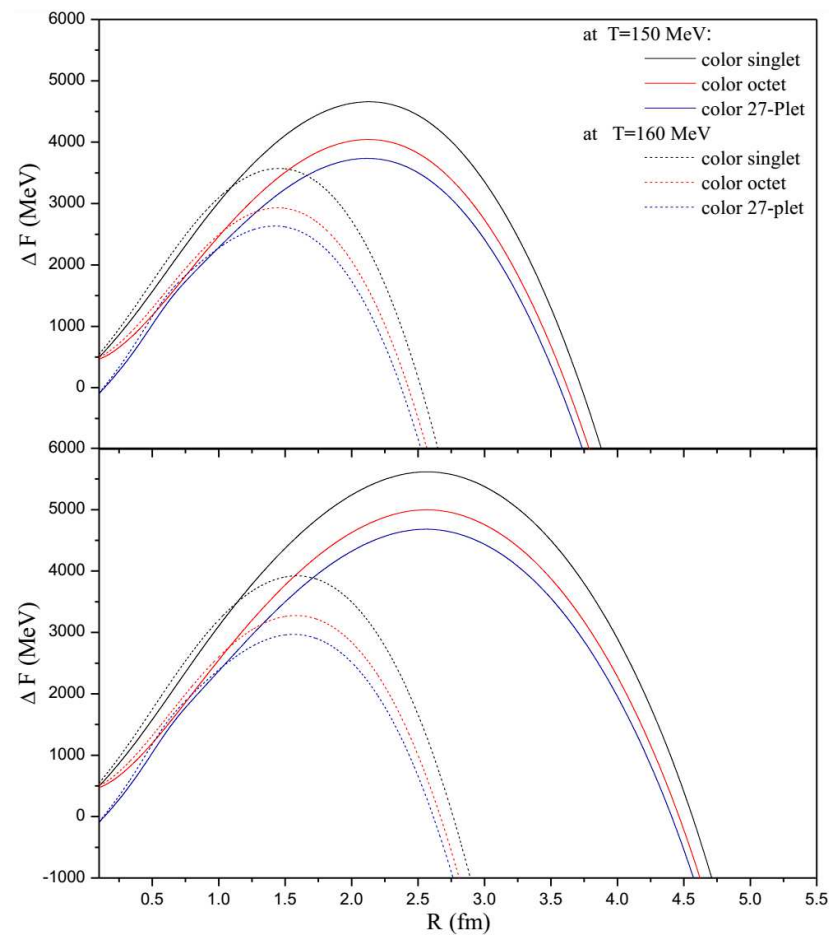

Fig. 4. Change in free energy vs the radius of QGP droplet with three quark flavors (top) and with two quark flavors (bottom), at two temperatures: $T=$ $150 \mathrm{MeV}$ (solid lines) and $T=160 \mathrm{MeV}$ (dashed lines), with $\sigma=20 \mathrm{MeV} / \mathrm{fm}^{2}$, in the three color representations: color singlet, color octet, color 27-plet.

The dependence on temperature of the radius of the QGP bubble, maximizing $\Delta F$, is visible from the curves, since at a fixed color representation of the QGP, the radius corresponding to a maximum of $\Delta F$ decreases with increasing temperature.

Another feature which can be drawn from all illustrated figures is that for small radii of the QGP droplet, 
the change in free energy for the same QGP color representation is constant and does not depend neither on the number of quark flavors, nor on the value of the surface tension, nor on temperature.

\section{Conclusion}

In the present work, we have studied the behavior of the free energy barrier with the radius of the QGP droplet formed in the nucleation process from a hadronic gas, for the three representations of the QGP: color singlet, color octet and color 27-plet, at different temperatures and for different numbers of quarks flavors, as well as for different surface tensions of the interface between the QGP and hadronic phases. We have found that the nucleated QGP bubble in the color octet or color 27-plet representations is always smaller than the color singlet droplet when formed. This result is obtained for both QGP with $u, d$ quarks and with $u, d$ and $s$ quarks, and for each color representation, the radius of the nucleated droplet of the QGP obtained with imposing the surface tension in the bag model is higher than the radius of the nucleated droplet of the QGP obtained at vanishing surface tension. Thus, the nucleation process does depend on the color representation, on the temperature, as well as on the number of quark flavors and on the surface tension.

\section{References}

[1] J.I. Kapusta, A.P. Vischer, R. Venugopalan, Phys. Rev. C 51, 901 (1995).

[2] I. Mardor, B. Svetitsky, Phys. Rev. D 44, 878 (1991).

[3] S.S. Singh, D.S. Gosain, Y. Kumar, A.K. Jha, Pramana-J. Phys 74, 27 (2010).

[4] D.S. Gosain, S.S. Singh, A.K. Jha, Pramana-J. Phys 78, 719 (2012).

[5] R. Becker, W. Döring, Ann. Phys. 416, 719 (1935).

[6] J. Frenkel, J. Chem. Phys. 7, 200 (1939).

[7] K. Redlich, L. Turko, Z. Phys. C 5, 201 (1980); L. Turko, Phys. Lett. B 104, 153 (1981).

[8] M. Ladrem, A. Ait El Djoudi, Eur. Phys. J. C 44, 257 (2005).

[9] A. Ait El Djoudi, Canad. J. Phys. 91, 793 (2013).

[10] W. Greiner, B. Müller, Quantum Mechanics — Symmetries, Springer-Verlag, 1994.

[11] H.-Th. Elze, W. Greiner, J. Rafelski, Phys. Lett. B 124, 515 (1983). 